\title{
Characterization of oil from roasted hemp seeds using the PDSC and FTIR techniques
}

\author{
Andrzej Bryśs (1D - Joanna Bryś ${ }^{2} \cdot$ Álvaro Fernández Mellado $^{3} \cdot$ Szymon Głowacki $^{1} \cdot$ Weronika Tulej $^{1}$ • \\ Ewa Ostrowska-Ligęza ${ }^{2} \cdot$ Piotr Koczoń $^{2}$
}

Received: 5 December 2018/Accepted: 25 July 2019/Published online: 10 August 2019

(C) The Author(s) 2019

\begin{abstract}
The purpose of the present work was to characterize oil extracted from roasted hemp seeds using the PDSC and FTIR techniques. Roasting was conducted in a laboratory convective dryer. DSC method was used for the determination of the oxidative stability of oil extracted from hemp seeds. Pressure differential scanning calorimetry experiments were carried out using a DSC Q20, TA Instruments, coupled with a high-pressure cell. The isothermal temperature (393 K) was used for the data collection. The oxidative induction time was obtained from the PDSC curves. Infrared spectra were registered for every sample in the classic range of $4000-400 \mathrm{~cm}^{-1}$ with $1 \mathrm{~cm}^{-1}$ resolution, using System 2000 PerkinElmer instrument. The Grams AI 8.0 and TQ Analyst 8 software were used for spectral data processing. The samples of oil were also characterized by standard quality parameters: fatty acids composition (with use of GC technique), free fatty acids content by titration with $0.1 \mathrm{M}$ potassium hydroxide and peroxide value determined by iodometric titration technique. Samples of oil extracted from hemp seeds after roasting in higher temperatures were characterized by lower induction time than oil from hemp seeds roasted in lower temperature. The results confirm that the induction time obtained from PDSC measurements can be used as parameters for the assessment of the resistance of oils from roasted hemp seeds to their thermaloxidative decomposition.
\end{abstract}

Keywords Hemp seed oil · Roasting process · PDSC · FTIR

\section{Introduction}

Roasted food products are one of the least processed products. The process of roasting products was used as early as in ancient times [1]. Seeds are roasted to improve their colour and organoleptic properties as well as extend storage life. Moreover, roasting leads to starch gelatinization and denaturation of proteins, thus improving digestibility of seeds. It is a simple and convenient process,

Andrzej Bryś

andrzej_brys@sggw.pl

1 Faculty of Production Engineering, Warsaw University of Life Sciences, Nowoursynowska 164, 02-776 Warsaw, Poland

2 Faculty of Food Sciences, Warsaw University of Life Sciences, Nowoursynowska 159c, 02-787 Warsaw, Poland

3 IES Pare Vitòria, Avinguda d'Elx 15, 03801 Alcoy, Alicante, Spain which involves exposure of the product to hot air for a short period of time [2, 3].

Hemp (Cannabis sativa L.) is one of the oldest plants cultivated in Europe and China (for thousands of years), used for preparing natural remedies, as a source of food, dietetic oil and cellulose. The content of oil in Cannabis sativa seeds amounts to approx. 35\% [4]. Hempseed oil is untraditional oil, which, as a source of essential unsaturated fatty acids and natural antioxidants, may be treated as a product for disease prevention and as a functional food. The main components of the oil are polyunsaturated fatty acids that belong to omega- 6 and omega- 3 acids family, i.e. linoleic acid (LA, 18: $2 \mathrm{n}-6$ ) and $\alpha$-linolenic acid (ALA, 18: $3 n-3)$. These acids are known as essential unsaturated fatty acids due to the fact that human organisms cannot produce them, and they must be supplemented with diet [5].

Oxidation is one of the most significant processes occurring in food which contains fat. Products of oxidation may have impact on other food components, leading to 
formation of desirable and undesirable compounds [6]. Cannabis sativa oil contains such bioactive compounds as tocopherols, polyphenols, phytosterols, carotenoids and other vitamins, which contribute to its high nutritional value. Compounds such as, i.a., sterols have impact on high antioxidative activity of the oil, i.e. inhibit the process of oxidation-related quality deterioration of the oil during storage. Moreover, due to their ability to bind free radicals, they are also useful in preventing such illnesses as cardiovascular disease, Alzheimer's disease and certain cancer types $[4,7]$. Phenol compounds present in hemp seed oil also contribute to increased oxidation stability of this oil, and have beneficial effect on human health (they prevent DNA, lipids and protein damage in human organism) [4, 8]. Beneficial effects of phytosterols in the oil are related to their activity that inhibits the absorption of cholesterol from food, which decreases the level of LDL cholesterol, and thus reduces the risk of cardiovascular disease $[4,9]$. The presence of carotenoids, including $\beta$ carotene (vitamin A provitamin) in hemp oil, may prevent colour change during storage. Undesirable components of hempseed oil include free fatty acids and chlorophylls, which act as pro-oxidants [4]. Due to the fact that oil obtained from hemp seeds is rich in polyunsaturated fatty acids, it may be susceptible to oxidization although it contains numerous antioxidative components. Lipids oxidation is generally considered a reaction that has an adverse effect on properties of edible oils and fats. Therefore, the main concern of producers is to ensure high quality of products of this type by monitoring the progress of oxidation process [6].

Oxygen molecule bonding with polyunsaturated fatty acids requires supply of energy. Therefore, oxidative stability of plant-derived oils may be determined by differential scanning calorimetry method (DSC) $[10,11]$. Recent research indicates that differential scanning calorimetry is an effective method for the assessment of plant-derived oil quality deterioration $[10,12-14]$. According to Kowalski et al. [15] high-pressure differential scanning calorimetry in isothermal conditions seems more appropriate for the assessment of edible oils oxidation than using DSC (without high pressure) as high-pressure DSC experiment is conducted in lower temperatures, for which the heat of transition may be determined more accurately.

Some researchers report $[16,17]$ that roasting increases stability of seeds by preventing self-oxidation of unsaturated fatty acids, increases its antioxidative activity and has a protective effect on DNA.

The goal of the present study was to investigate oxidative stability of oil extracted from roasted Cannabis sativa seeds by applying differential scanning calorimetry and Fourier-transform infrared spectroscopy (FTIR).

\section{Materials and methods}

Hemp seeds (BRAT.pl sp. z o.o., country of origin-the Netherlands) were roasted in the laboratory convective dryer (Memmert UFP400) in three temperatures: $393 \mathrm{~K}$, $423 \mathrm{~K}$ and $453 \mathrm{~K}$, for the period of 30 and $60 \mathrm{~min}$ at constant air flow rate of $\left(0.8-1.0 \mathrm{~m} \mathrm{~s}^{-1}\right)$.

Hexane was used to extract oil from the samples. Sizereduced seed samples with the mass of approx. $30 \mathrm{~g}$ were placed in conical flasks and mixed with $200 \mathrm{~mL}$ of hexane each. The mixture was shaken in a vortexer (vortex shaker) in water bath for $2 \mathrm{~h}$, followed by filtering the oil through a filter filled with anhydrous magnesium sulphate to distillation flasks. Vacuum evaporator was used to evaporate the solvent.

DSC method was used in order to determine oxidative stability of the oil extracted from hemp seeds. Experiments involving high-pressure differential scanning calorimetry were performed on a DSC Q20, TA Instruments, coupled with a high-pressure cell. Oil samples (3-4 mg) from seeds after roasting were placed in an aluminium pan, in oxygen atmosphere under the pressure of $1400 \mathrm{kPa}$. The measurement was taken isothermally at the temperature of 393 K. Oxidative induction time was determined from the PDSC curves.

Spectra for oil samples were registered in the transmission mode using two adjacent KRS plates, transparent for IR light with oil dropped between them to form a film. The measurement was conducted using PerkinElmer System 2000 spectrometer operated by Pergams software. The spectrum for each sample was registered in medium spectral range, i.e. $4000-400 \mathrm{~cm}^{-1}$. The resolution was set to $1 \mathrm{~cm}^{-1}$. Ten spectra (20 scans each) were recorded for each sample. Raw spectra were averaged and further processed with TQ Analyst 8 software.

Also, the content of free fatty acids was determined for the oil samples by $0.1 \mathrm{M}$ potassium hydroxide titration and the content of peroxides was determined by iodometric titration.

Fatty acids content was determined by gaseous chromatography using capillary column and flame-ionization detector (FID). In order to obtain volatile derivatives of fatty acids, the samples being examined underwent esterification by methanol, according to PN-EN ISO 5509:2000 norm, and fatty acids methyl esters were obtained. The prepared samples were injected onto the column. The test was performed with the YL6100 GC instrument equipped with BPX-70 capillary column with the length of $60 \mathrm{~m}$, internal diameter of $0.25 \mathrm{~mm}$ and film thickness of $0.25 \mu \mathrm{m}$, with nitrogen as carrier gas. The conditions of fatty acids methyl esters separation were as follows: initial temperature- $333 \mathrm{~K}, 5 \mathrm{~min}$; temperature from 333 to 
$453 \mathrm{~K}$ increased at a rate of $10 \mathrm{~K} \mathrm{~min}^{-1}$; temperature from 453 to $503 \mathrm{~K}$ increased at a rate $3 \mathrm{~K} \mathrm{~min}^{-1}$; final temperature $503 \mathrm{~K}$-for $15 \mathrm{~min}$, temperature of the detector was equal to $523 \mathrm{~K}$; and the temperature of the injector was equal to $498 \mathrm{~K}$; the total time of the analysis was equal to $51 \mathrm{~min}$. Fatty acids were identified based on their respective retention times, compared with the standard sample.

\section{Results and discussion}

Oil extracted from roasted hemp seeds contained significant amounts of polyunsaturated fatty acids (PUFA), amounting from 75.8 to $77.2 \%$ of all fatty acids (Fig. 1). The most important polyunsaturated acid in the analysed hemp oil samples was linoleic acid (52.4-52.8\%), belonging to omega- 6 acids family. Another important polyunsaturated acid was $\alpha$-linolenic acid (18.0-19.2\%) belonging to omega-3 acids family. The obtained oil also contained rare polyunsaturated $\gamma$-linolenic acid, amounting from 4.5 to $4.9 \%$. The ratio of omega- 6 to omega- 3 acids was approx. $3: 1$, which is very favourable from the nutritional point of view. The content of monounsaturated fatty acids (MUFA) in samples of oil extracted from roasted hemp seeds was between 11.1 and $11.9 \%$ of all fatty acids, and the content of saturated fatty acids (SFA) was between 9.6 and $10.4 \%$. The most important monounsaturated acid in the analysed samples of hempseed oil was oleic acid, with the content between 10.5 and $11.2 \%$. The main saturated acid in the oils obtained from roasted hemp seeds was palmitic acid, with the content between 5.7 and $6.8 \%$. The proportion of polyunsaturated fatty acids to saturated acids in the oil extracted from roasted hemp seeds was high (from 7.3 to 8.0). This coefficient is very favourable as oils with high content of unsaturated to saturated fatty acids have beneficial effect on human health by reducing the level of cholesterol in blood serum, thus preventing atherosclerosis and heart diseases [5, 18]. Similar content of fatty acids in hemp seed oil from unroasted seeds was obtained by other researchers. According to Porto et al. [5], the main components of the oil included the following acids: linoleic, $\alpha$-linolenic and oleic acid, with the total amount between 88.6 and $88.8 \%$, followed by palmitic acid $(5.2-5.4 \%)$ and $\gamma$-linolenic acid $(3.5 \%)$. Based on the performed experiments, it may be concluded that roasting did not have a significant impact on the content of fatty acids in the oil. Small differences in the composition of the fatty acids were obtained for oil extracted from hemp seeds roasted in the temperature of $453 \mathrm{~K}$ for $60 \mathrm{~min}$. The duration and high temperature of heat treatment reduced the content of polyunsaturated acids and increased the content of saturated acids, particularly, stearic acid.

The main components of fat are triacylglycerols. Apart from triacylglycerols, fat also contains some free fatty acids, diacylglycerols and monoacylglycerols. Increased content of non-triacylglycerols may result in lower resistance to oxidation, and in loss of fatty substance [19-21]. Figure 2 shows the content of free fatty acids in oil from roasted Cannabis sativa seeds. The content of free fatty acids in the samples was analysed, with no statistically significant differences or only small differences being observed. The content of free fatty acids decreased with the increase in temperature and duration of the process of seed roasting. The content of free fatty acids in the oils from
Fig. 1 Content of fatty acids (SFA-saturated fatty acids, MUFA-monounsaturated fatty acids, PUFA—polyunsaturated fatty acids) for oil extracted from roasted hemp seeds
घSFA $\square$ MUFA $\square$ PUFA $\square$ OTHER

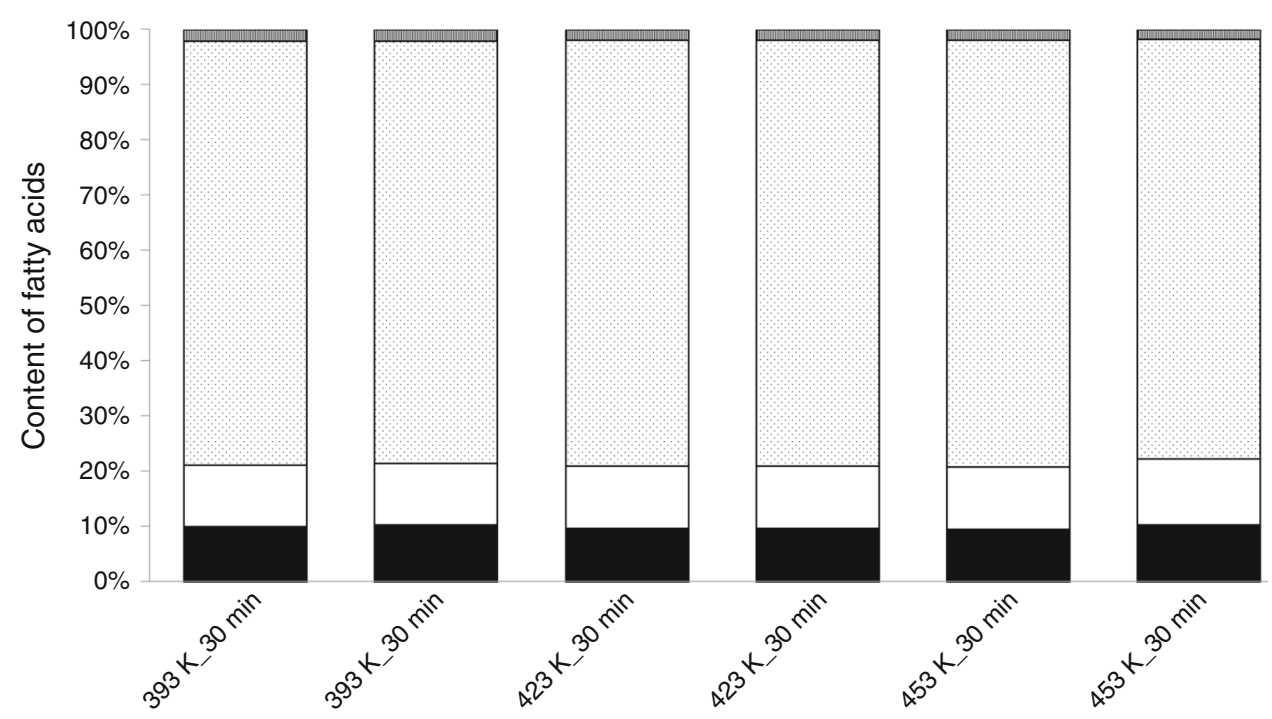




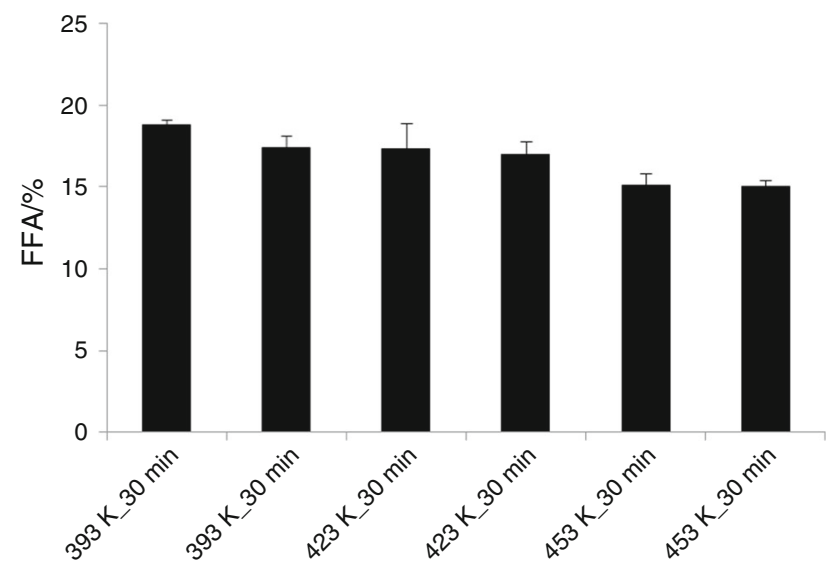

Fig. 2 Free fatty acids (FFA) content in oil from roasted hemp seeds

roasted hemp seeds was between 15.0 and $18.8 \%$. Roasting, therefore, did not have a significant impact on hydrolytic processes in oils.

Fat oxidation results in chemical changes leading to deterioration of taste, smell and food spoilage. Radicals, as intermediate products, may have adverse impact on human health. Factors favourable to oxidation include, i.a., light, oxygen and high temperature [22]. The most important factors that have impact on oxidation rate include fatty acids composition and the presence of pro-oxidants and antioxidants [23]. Oils, which contain polyunsaturated fatty acids, are the most susceptible to oxidation [22, 23]. DSC method, applied in this work, may be used to monitor kinetics of crystallization, determine thermodynamic parameters and determine the quality of lipids and oxidative stability [24-28]. In order to determine oxidative stability of fat, induction time was determined in the accelerated oxidation test. In fat stability tests, the rule is that oxidative stability of the fat increases with induction time [29].

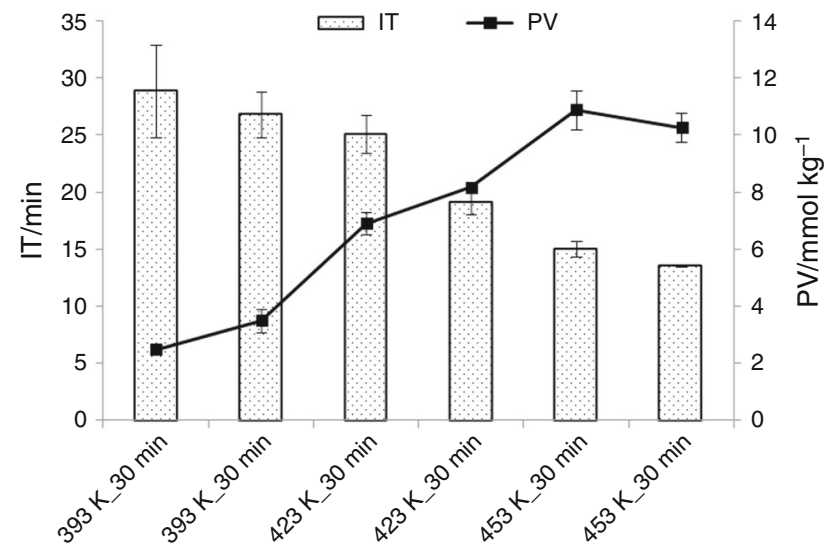

Fig. 3 Oxidation induction time (IT) and peroxide value (PV) of oil from roasted hemp seeds
Oxidation induction time for oils extracted from roasted hemp seeds is presented in Fig. 3. The time ranged from 13.6 to $28.9 \mathrm{~min}$. Oxidative stability for the obtained oils was compared with the results obtained for other plant oils, analysed by DSC method, in similar conditions. Ciemniewska-Żytkiewicz et al. [11], analysed cold-pressed oil from hazelnuts, first-pressed olive oil and refined rape oil. The obtained results were as follows: oxidation induction time for hazelnut oil, first-pressed olive oil and rape oil were equal 145,157 and $90 \mathrm{~min}$, respectively. In comparison with other oils, hempseed oil is extremely susceptible to oxidation, which may be attributed to high content of polyunsaturated fatty acids in the oil. Among the examined oils, the oil from hemp seeds roasted for $30 \mathrm{~min}$ at the temperature of $393 \mathrm{~K}$ was characterized by longer induction time, while the oil extracted from hemp seeds roasted for $60 \mathrm{~min}$ at the temperature of $453 \mathrm{~K}$ had the shortest induction time. Given the results, it may be concluded that higher temperature and longer time of roasting have adverse impact on oxidative stability of the oil.

Figure 3 also shows changes in the value of the peroxide number versus roasting conditions. Peroxide number is indicative of the initial degree of unsaturated fatty acids oxidation. According to the values defined in the Codex Alimentarius [30], the value of the peroxide number for refined oils should not exceed $10 \mathrm{mEq} \mathrm{O} \mathrm{kg}^{-1}$ $\left(5 \mathrm{mmol} \mathrm{O}_{2} \mathrm{~kg}^{-1}\right)$, and for unrefined oils- $15 \mathrm{mEq} \mathrm{O}_{2}$ $\mathrm{kg}^{-1}\left(7.5 \mathrm{mmol} \mathrm{O}_{2} \mathrm{~kg}^{-1}\right)$. Given the obtained results, it may be concluded that the content of initial oxidation products is significantly (over fourfold) higher in oil from seeds roasted for a longer period of time at higher temperature in comparison with the content of these components in oil from seeds roasted for a shorter period of time at lower temperature. The peroxide number for oil from seeds roasted at $453 \mathrm{~K}$ and at $423 \mathrm{~K}$ for $60 \mathrm{~min}$ was between 8.2 and $10.9 \mathrm{mmol} \mathrm{O}_{2} \mathrm{~kg}^{-1}$, and the obtained values exceeded the maximum acceptable value defined in the Codex Alimentarius. Peroxide numbers obtained for other examined oils were within the norm and ranged between 2.5 and $6.9 \mathrm{mmol} \mathrm{O}_{2} \mathrm{~kg}^{-1}$.

The results of oxidative stability tests performed by DSC method and titrimetric method show the inverse relationship between peroxide number and induction time. Induction time is inversely proportional to the content of initial oxidation products. The obtained results are consistent with other researches [31].

Spectra registered are presented in Fig. 4. The visual inspection proves high similarity of all spectra. However, in the region of 1500-1350 some distinct differences clearly occur in wavelengths and intensities of bands in the spectra of differently treated samples, e.g. different times of roasting and different temperatures applied. The changes 


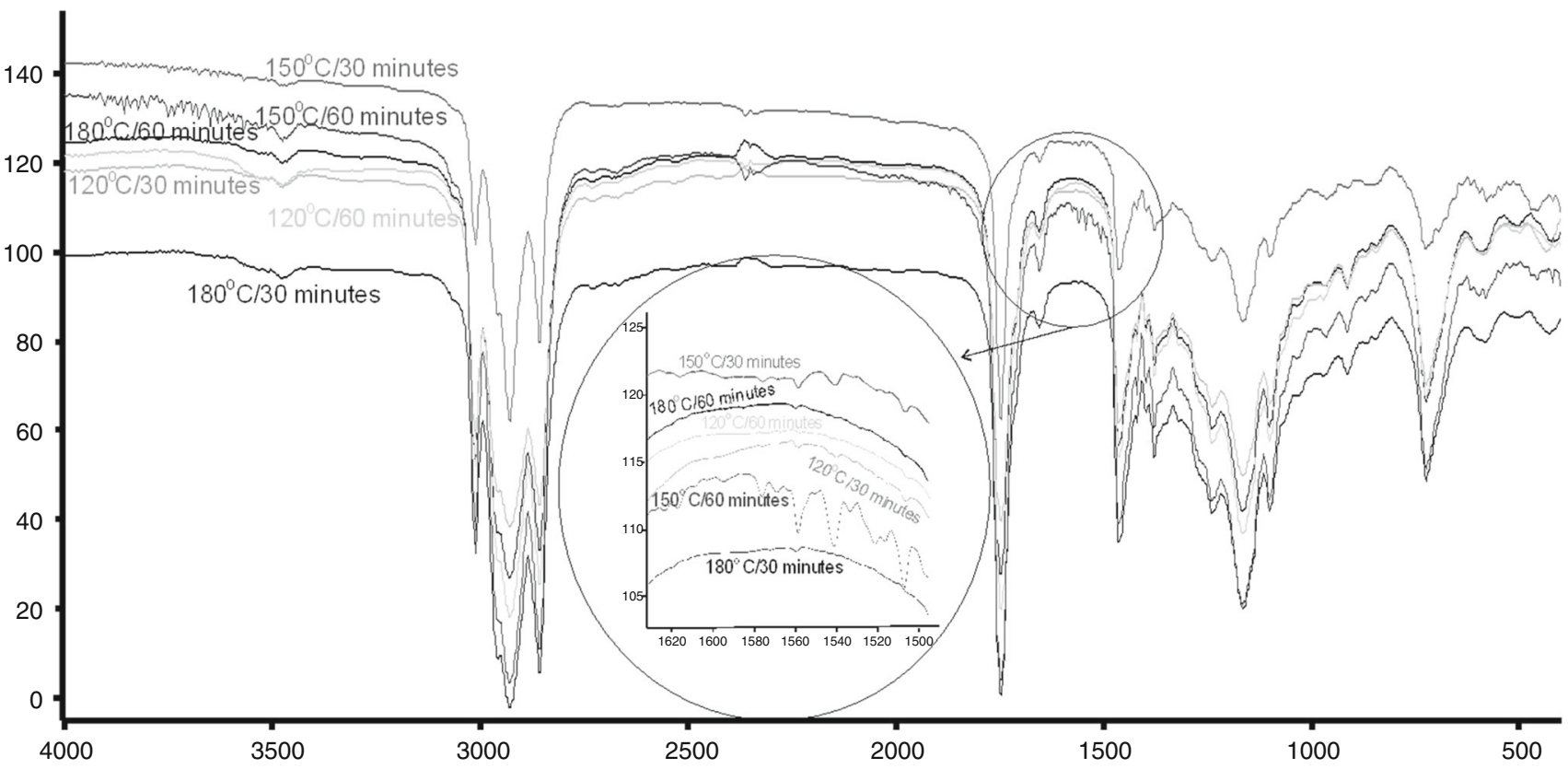

Fig. 4 FTIR spectra of oils from hemp seeds roasted in $393 \mathrm{~K}\left(120^{\circ} \mathrm{C}\right), 423 \mathrm{~K}\left(150{ }^{\circ} \mathrm{C}\right)$ or $453 \mathrm{~K}\left(180{ }^{\circ} \mathrm{C}\right)$ for 30 or 60 min, respectively. Circle magnifies region with distinct differences identified with visual inspection

in spectral data are due to changes in chemical composition of samples. They are present in different spectral regions and difficult to follow as single compound can increase overall intensity in one region and decrease in other. It can result in change in intensity and appearing and disappearing of specific bands. As studied samples are physically homogenous mixtures of many different chemicals, small changes can occur in the same time in each mixture component, which results in unexpected and unpredictable spectral changes. Those changes can be, however, statistically related to the amount of given component or to a value of feature that results from the presence of this component or components [20,32,33]. The statistical procedure of PLS (partially least square) resulted in finding statistical models correlating peroxide value, induction time and free fatty acids content with spectral data, respectively. For all models, two spectral regions were selected: $3060-2782$ and $1870-1386 \mathrm{~cm}^{-1}$. The calibration and validation sets were randomly selected by software. The features of the best model calculated for peroxide value (MODEL_1) were as follows $R^{2}=0.9840$, and RMSEC $=0.626$ with the prediction $R^{2}=0.9664$ and RMSEP $=1.99$. Five factors were used to produce the model. For induction time model (MODEL_2), features were as follows: $R^{2}=0.9954$, and RMSEC $=0.595$ for calibration, and $R^{2}=0.9659$ and RMSEP $=2.67$ for prediction. Five factors were used to produce the model. For MODEL_3, i.e. free fatty acids content-spectral data statistical features were as follows: $R^{2}=0.9817$, and RMSEC $=0.257$ for calibration, and $R^{2}=0.8997$ and
RMSEP $=0.496$ for prediction. Five factors were used to calibrate the model.

\section{Conclusions}

1. Oil from roasted hemp seeds contained considerable amount of polyunsaturated fatty acids. It also had a high proportion of polyunsaturated fatty acids to saturated acids, and favourable, from the nutritional point of view, proportion of omega- 6 to omega- 3 acids.

2. The process of roasting did not have a significant impact on the content of fatty acids. Small differences between the compositions of fatty acids may be observed for oil extracted from seeds roasted at $453 \mathrm{~K}$ for $60 \mathrm{~min}$.

3. Roasting at higher temperature and for longer periods of time results in shorter induction time and higher peroxide number of oils from roasted hemp seeds, and, consequently, in worse oxidative stability in comparison with oils from seeds roasted in lower temperature for a shorter period of time.

4. Among the examined oils, the oil from seeds roasted at $393 \mathrm{~K}$ for $30 \mathrm{~min}$ was characterized by the best oxidative stability. Three standard features related to oil quality, i.e. peroxide value, induction time and free fatty acids content, can be determined by IR spectroscopy once sensitive spectral region has been found.

This paper belongs to the ESTAC12 Conference. 
Open Access This article is distributed under the terms of the Creative Commons Attribution 4.0 International License (http://creative commons.org/licenses/by/4.0/), which permits unrestricted use, distribution, and reproduction in any medium, provided you give appropriate credit to the original author(s) and the source, provide a link to the Creative Commons license, and indicate if changes were made.

\section{References}

1. Baba WN, Rashid I, Shah A, Ahmad M, Gani A, Masoodi FA, Wani IA, Wani SM. Effect of microwave roasting on antioxidant and anticancerous activities of barley flour. J Saudi Soc Agric Sci. 2016;15:12-9.

2. Venkatesh Murthy K, Ravi R, Keshava Bhat K, Raghavarao KSMS. Studies on roasting of wheat using fluidized bed roaster. J Food Eng. 2008;89:336-42.

3. Sharma P, Singh Gujral H, Rosell CM. Effects of roasting on barley b-glucan, thermal, textural and pasting properties. J Cereal Sci. 2011:53:25-30.

4. Liang J, Aachary A, Thiyam-Holländer U. Hemp seed oil: minor components and oil quality. Lipid Tech. 2015;27(10):231-3.

5. Porto C, Decorti D, Tubaro F. Fatty acid composition and oxidation stability of hemp (Cannabis sativa L.) seed oil extracted by supercritical carbon dioxide. Ind Crop Prod. 2012;36:401-4.

6. Wąsowicz E, Gramza A, Hęś M, Jeleń HH, Korczak J, Małecka M, Mildner-Szkudlarz S, Rudzińska M, Samotyja U, ZawirskaWojtasiak R. Oxidation of lipids in food. Pol J Food Nutr Sci. 2004;13(54):87-100.

7. Matthäus B, Brühl L. Virgin hempseed oil: an interesting niche product. Eur J Lipid Sci Technol. 2008;110:655-61.

8. Siger A, Nogala-Kalucka M, Lampart-Szczapa E. The content and antioxidant activity of phenolic compounds in cold-pressed plant oils. J Food Lipid. 2008;15:137-49.

9. Ghazani SM, Marangoni AG. Minor components in canola oil and effects of refining on these constituents: a review. J Am Oil Chem Soc. 2013;90:923-32.

10. Tan CP, Che Man YB. Recent developments in differential scanning calorimetry for assessing oxidative deterioration of vegetable oils. Trends Food Sci Technol. 2002;13:312-8.

11. Ciemniewska-Żytkiewicz H, Ratusz K, Bryś J, Reder M, Koczon P. Determination of the oxidative stability of hazelnut oils by PDSC and Rancimat methods. J Therm Anal Calorim. 2014;118:875-81.

12. Ostrowska-Ligęza E, Górska A, Wirkowska M, Koczoń P. An assessment of various powdered baby formulas by conventional methods (DSC) or FT-IR spectroscopy. J Therm Anal Calorim. 2012;110:465-71.

13. Górska A, Ostrowska-Ligęza E, Szulc K, Wirkowska M. A differential scanning calorimetric study of $\beta$-lactoglobulin and vitamin D3 complexes. J Therm Anal Calorim. 2012;110:473-7.

14. Górska A, Szulc K, Ostrowska-Ligęza E, Wirkowska M, Bryś J. The influence of trehalose-maltodextrin and lactose-maltodextrin matrices on thermal and sorption properties of spray-dried $\beta$ lactoglobulin-vitamin D3 complexes. J Therm Anal Calorim. 2013;112:429-36.

15. Kowalski B, Gruczyńska E, Maciaszek K. Kinetics of rapeseed oil oxidation by pressure differential scanning calorimetry measurements. Eur J Lipid Sci Technol. 2000;102:337-41.

16. Krings U, Berger RG. Antioxidant activity of some roasted foods. Food Chem. 2001;72:223-9.

17. Gelmeza N, Kincala NS, Yenerb ME. Optimization of supercritical carbon dioxide extraction of antioxidants from roasted wheat germ based on yield, total phenolic and tocopherol contents, and antioxidant activities of the extracts. J Supercrit Fluids. 2009;48:217-24.

18. Rudel LL, Kelley K, Sawyer JK, Shah R, Wilson MD. Dietary monounsaturated fatty acids promote aortic atherosclerosis in LDL receptor-null, human ApoB100-overexpressing transgenic mice. Arterioscler Thromb Vasc Biol. 1998;18:1818-27.

19. Martin D, Reglero G, Senorans FJ. Oxidative stability of structured lipids. Eur Food Res Technol. 2010;231:635-53.

20. Bryś J, Wirkowska M, Górska A, Ostrowska-Ligęza E, Bryś A, Koczoń P. The use of DSC and FT-IR spectroscopy for evaluation of oxidative stability of interesterified fats. J Therm Anal Calorim. 2013;113:481-7.

21. Bryś J, Flores IF, Górska A, Wirkowska-Wojdyła M, OstrowskaLigęza E, Bryś A. Use of GC and PDSC methods to characterize human milk fat substitutes obtained from lard and milk thistle oil mixtures. J Therm Anal Calorim. 2017;130:319-27.

22. Thurgood J, Ward R, Martini S. Oxidation kinetics of soybean oil/anhydrous milk fat blends: a differential scanning calorimetry study. Food Res Int. 2007;40:1030-7.

23. Yankah VV, Akoh CC. Batch enzymatic synthesis, characterization and oxidative stability of DHA-containing structured lipids. J Food Lipids. 2000;7:247-61.

24. Reamy A, Lambelet P, Rousset P. Calorimetric information about food and food constituents. In: Lörinczy D, editor. The nature of biological systems as revealed by thermal methods. Dordrecht: Kluwer Academic Publishers; 2004. p. 69-98.

25. Vicente J, Cappato LP, Araújo Calado VM, Carvalho MG, Garcia-Rojas EE. Thermal and oxidative stability of Sacha inchi oil and capsules formed with biopolymers analyzed by DSC and ${ }^{1} \mathrm{H}$ NMR. J Therm Anal Calorim. 2018;131:2093-104.

26. Fontanari GG, Kobelnik M, Marques MR, Gomes Areas JA, Trevizan Franzin B, Aparecida Pastre I, Fertonani LF. Thermal and kinetic studies of white lupin (Lupinus albus) oil. J Therm Anal Calorim. 2018;131:775-82.

27. Kobelnik M, Fontanari GG, Ribeiro CA, Spirandeli Crespi M. Evaluation of thermal behavior and chromatographic characterization of oil extracted from seed of Pittosporum undulatum. J Therm Anal Calorim. 2018;131:371-8.

28. Fonseca M, Ferreira LMB, Soares RAM, Kobelnik M, Fontanari GG, Crespi MS, Ribeiro CA. Extraction of soursop oil (Annona muricata L.) by ultrasonic technique Chromatographic evaluation and thermal characterization. $\mathrm{J}$ Therm Anal Calorim. 2018;134:1893-901.

29. Kowalski B, Ratusz K, Kowalska D, Bekas W. Determination of the oxidative stability of vegetable oils by differential scanning calorimetry and Rancimat measurements. Eur J Lipid Sci Technol. 2004;106:165-9.

30. Standard for named vegetable oils. Codex Alimentarius. 2001; Codex Stan 210-1999.

31. Bryś J, Wirkowska M, Górska A, Ostrowska-Ligęza E, Bryś A. Application of the calorimetric and spectroscopic methods in analytical evaluation of the human milk fat substitutes. J Therm Anal Calorim. 2014;118:841-8.

32. Koczoń P, Lipińska E, Czerniakowska-Piątkowska E, Mikuła M, Bartyzel BJ. The changes of fatty acids composition of Polish biscuits during storage. Food Chem. 2016;202:341-8.

33. Sujka K, Koczon P, Ceglińska A, Reder M, CiemniewskaŻytkiewicz H, Koczoń P. The application of FT-IR spectroscopy for quality control of flours obtained from polish producers. J Anal Methods Chem. 2017;2017:4315678.

Publisher's Note Springer Nature remains neutral with regard to jurisdictional claims in published maps and institutional affiliations. 